\title{
LINEARIZATION OF HOMEOMORPHISM GROUPS
}

\author{
SU-SHING CHEN
}

ABSTRACT. A theorem of Beboutov and Kakutani is extended to a large class of homeomorphism groups.

Let $G$ be a group of homeomorphisms of a metric space $X$. We are concerned with the question of linearization of the action of $G$ [6], [7]. More specifically, we ask the question whether the given action $\phi: G \times X \rightarrow X$ of $G$ on $X$ can be embedded in the natural action $\pi: G \times C^{*}(G) \rightarrow C^{*}(G)$ of $G$ on the Banach algebra $C^{*}(G)$ of bounded continuous functions on $G$ with the sup norm, defined by $\pi(g, f)\left(g^{\prime}\right)=f\left(g g^{\prime}\right), g, g^{\prime} \in G$ and $f \in C^{*}(G)$. When $G$ is a 1-parameter group of homeomorphisms of a compact metric space $X$, this is answered by Beboutov and Kakutani [4]. We shall extend their theorem to a large class of homeomorphism groups (not necessarily finite dimensional). This class contains connected Lie groups [8] and connected Banach Lie groups. It is desirable to consider infinite dimensional groups of homeomorphisms, because the whole homeomorphism groups of metric spaces are mostly infinite dimensional.

For any locally compact space $X$, the modified compact-open topology for a group $G$ of homeomorphisms of $X$ implies that the 1-parameter subgroups of $G$ are exactly the actions of the real numbers $\mathbf{R}$ on $X$ whose ranges lie in $G$ [2]. From now on, we shall always use this topology. Consider the set $\operatorname{Hom}(\mathbf{R}, G)$ of all 1-parameter subgroups of $G$. Then this set has a scalar multiplication in the following manner. For $s, t$ in $\mathbf{R}$ and $\lambda$ in $\operatorname{Hom}(\mathbf{R}, G),(s \cdot \lambda)(t)=\lambda(s t)$, such that $0 \cdot \lambda=0 \cdot \mu$ for all $\lambda$, $\mu$ in $\operatorname{Hom}(\mathbf{R}, G)$, and $1 \cdot \lambda=\lambda$ for all $\lambda$ in $\operatorname{Hom}(\mathbf{R}, G)$. Let exp: $\operatorname{Hom}(\mathbf{R}, G) \rightarrow G$ be the evaluation map at 1 in $\mathbf{R}$. A transformation group $(G, X, \phi)$ is called a generalized Lie transformation group if $\operatorname{Hom}(\mathbf{R}, G)$ can be given the structure of a real locally convex topological Lie algebra such that (1) the scalar multiplication of $\operatorname{Hom}(\mathbf{R}, G)$ is the scalar multiplication of the Lie algebra, and (2) the map exp: $\operatorname{Hom}(\mathbf{R}, G) \rightarrow G$ is continuous and it maps a neighborhood of 0 in $\operatorname{Hom}(\mathbf{R}, G)$ onto a neighborhood of identity of $G$. Clearly generalized Lie transformation groups possess a lot of 1-parameter subgroups. Lie transformation groups and Banach Lie transformation groups are generalized Lie transformation groups. In fact, the Lie algebra $\operatorname{Hom}(\mathbf{R}, G)$ of $G$ has an open ball of 0 homeomorphic to a neighborhood of identity of $G$.

Received by the editors June 1, 1974 and, in revised form, July 24, 1974. AMS (MOS) subject classifications (1970). Primary 57E05.

Key words and phrases. Homeomorphism, 1-parameter subgroup. 
The main theorem can be stated as follows.

Theorem. Let $(G, X, \phi)$ be a connected generalized Lie transformation group of a compact metric space $X$. There exists a G-equivariant homeomorphism of $X$ into the Banach algebra $C^{*}(G)$ of all bounded continuous functions if and only if the fixed point set of $G$ in $X$ is either empty or homeomorphic to a subset of the real numbers $\mathbf{R}$.

It is easy to see that the condition of the Theorem is necessary. The fixed point set of $G$ in $C^{*}(G)$ consists of exactly all constant functions; and hence is homeomorphic to the real numbers $R$. If there is a $G$-equivariant homeomorphism of $X$ into $C^{*}(G)$, the fixed point set $F$ of $G$ in $X$ must be homeomorphic to a subset of the real numbers $R$.

In the sequel, we shall show the condition is also sufficient. Let $\gamma$ be a real-valued continuous function defined on $F$ which gives the homeomorphism of $F$ into a subset $\gamma(F)$ of $\mathbf{R}$. Let $C(X)$ be the Banach algebra of all continuous functions $f$ on $X$ with the supremum norm. Then $C(X)$ is a separable metric space. Let

$$
\Phi=C(X, F, \gamma)=\{f \mid f \in C(X), f(x)=\gamma(x) \text { for any } x \in F\} .
$$

Then $\Phi$ is a nonempty subset of $C(X)$ by Tietze's extension theorem, and a closed subset of $C(X)$, and hence a complete, separable metric space with respect to the metric $d(f, g)=\|f-g\|$.

Lemma 1. Under the assumption of the above Theorem, there exists a function $f \in \Phi$ such that for any two points $x, y \in X$, with $x \neq y$, there exists an element $g \in G$ such that $f(\phi(g, x)) \neq f(\phi(g, y))$.

Let us assume Lemma 1 is true. For any point $x \in X$, let us define a bounded continuous function $x^{*}$ on $G$ by $x^{*}(g)=f(\phi(g, x))$. Let $\sigma$ be a mapping of $X$ into $C^{*}(G)$ defined by $\sigma: x \rightarrow x^{*}$. Then $\sigma$ is a one-to-one continuous mapping of $X$ into $C^{*}(G)$. Since $X$ is compact, $\sigma$ is a homeomorphism of $X$ onto a subset $\sigma(X)$ of $C^{*}(G)$. Moreover $\sigma(\phi(g, x))=\pi(g, \sigma(x))$, for any $x \in X$ and $g \in G$. Thus we have proved the Theorem.

Consider the Cartesian products $X^{*}=X \times X$ and $F^{*}=F \times F$ and a subset $A^{*}$ of $X^{*}$ defined by $A^{*}=X^{*}-\left(F^{*} \cup \Delta^{*}\right)$ where $\Delta^{*}$ is the diagonal of $X^{*} . X^{*}$ is a compact metric space and $A^{*}$ is an open subset of $X^{*}$. For any compact subset $K^{*}$ of $A^{*}$ we denote by $\Phi\left(K^{*}\right)$ the set of all $f \in \Phi$ such that, for any two points $x, y \in X$ with $(x, y) \in K^{*}$, there exists an element $g \in G$ such that $f(\phi(g, x)) \neq f(\phi(g, y))$.

Lemma 2. $\Phi\left(K^{*}\right)$ is an open subset of $\Phi$ for any compact subset $K^{*}$ of $A^{*}$. 
Proof. Assume $K^{*}$ is a compact subset of $A^{*}$ and let $f_{0}$ be a function from $\Phi\left(K^{*}\right)$. Let

$$
\rho(x, y)=\sup _{g \in G}\left|f_{0}(\phi(g, x))-f_{0}(\phi(g, y))\right| .
$$

Then $\rho(x, y)>0$ for any $(x, y) \in K^{*}$. There exists a positive number $\delta_{0}>0$ such that $\rho(x, y) \geq \delta_{0}$. for any $(x, y) \in K^{*}$. In, fact, if this is not true, then there is a sequence $\left\{\left(x_{n}, y_{n}\right) \mid n=1,2, \ldots\right\}$ of elements from $K^{*}$ such that $\rho\left(x_{n}, y_{n}\right) \rightarrow 0$. Since $K^{*}$ is compact, we may assume that there exists an element $\left(x_{0}, y_{0}\right) \in K^{*}$ such that $x_{n} \rightarrow x_{0}$ and $y_{n} \rightarrow y_{0}$. Thus $f_{0}\left(\phi\left(g, x_{n}\right)\right) \rightarrow$ $f_{0}\left(\phi\left(g, x_{0}\right)\right), f_{0}\left(\phi\left(g, y_{n}\right)\right) \rightarrow f_{0}\left(\phi\left(g, y_{0}\right)\right)$ for each $g \in G$. Since

$$
\left|f_{0}\left(\phi\left(g, x_{n}\right)\right)-f_{0}\left(\phi\left(g, y_{n}\right)\right)\right| \leq \rho\left(x_{n}, y_{n}\right)
$$

for each $g \in G$. This contradicts that $\rho\left(x_{0}, y_{0}\right)>0$. Thus, there exists a positive number $\delta_{0}>0$ such that $\rho(x, y) \geq \delta_{0}$ for any $(x, y) \in K^{*}$. Thus $f \in \Phi$ and $\left\|f-f_{0}\right\|<\delta_{0} / 2$ imply that $f \in \Phi\left(K^{*}\right)$ is an open subset of $\Phi$.

Lemma 3. For any two points $x, y \in X$ with $(x, y) \in A^{*}$, there exists a compact neighborhood $K^{*}$ of $(x, y)$ in $A^{*}$ such that $\Phi\left(K^{*}\right)$ is dense in $\Phi$.

Proof. Let $\left(x_{0}, y_{0}\right) \in A^{*}$ such that $x_{0} \in X-F, y_{0} \in X$ and $x_{0} \neq y_{0}$. Let $V$ be an open neighborhood of $x_{0}$ such that $F \subset X-\bar{V}$ and $y_{0} \in X-\bar{V}$, where $\bar{V}$ denotes the closure of $V$ in $X$. Since $x_{0}$ is not a fixed point of $G$ in $X$, there exists an element $g_{0}$ in a small neighborhood of identity of $G$ such that $\phi\left(g_{0}, x_{0}\right) \neq x_{0}$. Then there exists a 1 -parameter subgroup $\lambda$ of $G$ such that $g_{0}=\lambda(\eta)$ for a positive number $\eta>0$ so small that $\operatorname{arc}\left(x_{0},[0, \eta]\right)$ and tube $(X-V,[0, \eta])$ are disjoint (see [4] for notation). Now we can apply the argument in [4] to the 1-parameter subgroup $\dot{\lambda}$ to show that there exists a compact neighborhood $K^{*}$ of $\left(x_{0}, y_{0}\right)$ in $A^{*}$ such that $\Phi\left(K^{*}\right)$ is dense in $\Phi$.

Finally we prove Lemma 1 . Since $A^{*}$ is a separable metric space, there exists a sequence $\left\{K_{n}^{*} \mid n=1,2, \ldots\right\}$ of compact subsets $K_{n}^{*}$ of $A^{*}$ such that each $\Phi\left(K_{n}^{*}\right)$ is dense in $\Phi$ and $A^{*}=\bigcup_{n=1}^{\infty} K_{n}^{*}$. Since each $\Phi\left(K_{n}^{*}\right)$ is an open subset of $\Phi$, and since $\Phi$ is a complete metric space, the intersection $\bigcap_{n=1}^{\infty} \Phi\left(K_{n}^{*}\right)$ is not empty. Thus, any function $f$ from $\bigcap_{n=1}^{\infty} \Phi\left(K_{n}^{*}\right)$ satisfies the condition of Lemma 1.

\section{REFERENCES}

1. S. Chen and R. Yoh, The category of generalized Lie groups, Trans. Amer. Math. Soc. 199 (1974), 281-294.

2. A. M. Gleason and R. S. Palais, On a class of transformation groups, Amer. J. Math. 79 (1957), 631-648. MR 19, 663.

3. K. Hofmann, Introduction to the theory of compact groups, Tulane University Lecture Notes, 1968. 
4. S. Kakutani, A proof of Beboutov's theorem, J. Differential Equations 4 (1968), 194-201. MR 37 \# 1734.

5. D. Montgomery and L. Zippin, Topological transformation groups, Interscience, New York and London, 1955. MR 17, 383.

6. G. D. Mostow, Equivariant embeddings in Euclidean space, Ann. of Math. (2) 65 (1957), 432-446. MR 19, 291.

7. - On a conjecture of Montgomery, Ann. of Math. (2) 65 (1957), 513516. MR 19, 292.

8. S. Chen, An extension of the Kakutani-Beboutov system, J. Differential Equations 18 (1975).

DEP ARTMENT OF MATHEMATICS, UNIVERSITY OF FLORIDA, GAINSVILLE, FLORIDA 32611 